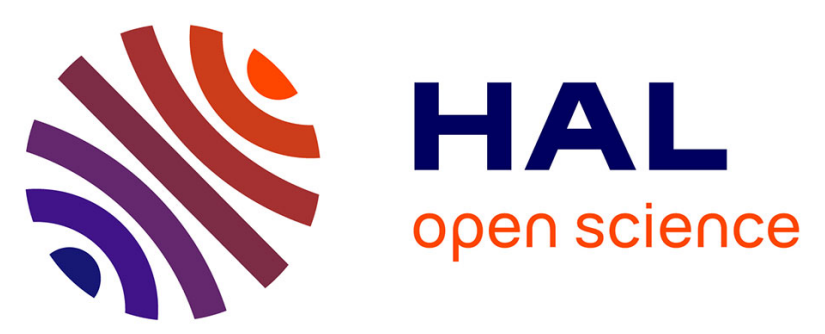

\title{
Ferroelectric HfO2 for Memory Applications: Impact of Si Doping Technique and Bias Pulse Engineering on Switching Performance
}

T. Francois, J. Coignus, L. Grenouillet, J.P. P Barnes, N. Vaxelaire, J. Ferrand, I. Bottala-Gambetta, M. Gros-Jean, S. Jeannot, P. Boivin, et al.

\section{To cite this version:}

T. Francois, J. Coignus, L. Grenouillet, J.P. P Barnes, N. Vaxelaire, et al.. Ferroelectric HfO2 for Memory Applications: Impact of Si Doping Technique and Bias Pulse Engineering on Switching Performance. 2019 IEEE 11th International Memory Workshop (IMW), May 2019, Monterey, United States. pp.1-4, 10.1109/IMW.2019.8739664 . hal-02399691

\section{HAL Id: hal-02399691 https://hal.science/hal-02399691}

Submitted on 9 Dec 2019

HAL is a multi-disciplinary open access archive for the deposit and dissemination of scientific research documents, whether they are published or not. The documents may come from teaching and research institutions in France or abroad, or from public or private research centers.
L'archive ouverte pluridisciplinaire HAL, est destinée au dépôt et à la diffusion de documents scientifiques de niveau recherche, publiés ou non, émanant des établissements d'enseignement et de recherche français ou étrangers, des laboratoires publics ou privés. 


\title{
Ferroelectric $\mathrm{HfO}_{2}$ for Memory Applications: Impact of Si Doping Technique and Bias Pulse Engineering on Switching Performance
}

\author{
T. Francois ${ }^{1,2}$, J. Coignus ${ }^{1}$, L. Grenouillet ${ }^{1}$, J.P. Barnes ${ }^{1}$, N. Vaxelaire ${ }^{1}$, J. Ferrand ${ }^{3}$, I. Bottala-Gambetta ${ }^{3}$, M. Gros-

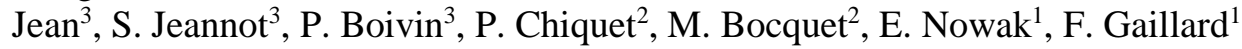 \\ ${ }^{1}$ Université Grenoble-Alpes, CEA-Leti, Grenoble, France, ${ }^{2}$ Aix Marseille Univ, Université de Toulon, CNRS, IM2NP, Marseille, \\ France, ${ }^{3}$ STMicroelectronics, Crolles, F-38920, France. \\ * corresponding author: terry.francois@cea.fr
}

\begin{abstract}
A clear comparison between Atomic Layer Deposition and Ion Implantation $\mathrm{Si}$ doping techniques is established. Comparable remnant polarization and coercive fields are obtained at lower $\mathrm{Si}$ content $(\% \mathrm{Si})$ for Ion Implantation, with a slight decrease of endurance performance. Switching signal engineering demonstrates a wide range of performance achievable with $\mathrm{HfO}_{2}: \mathrm{Si}$ ferroelectric layer.
\end{abstract}

Keywords-component; memory; $\mathrm{FeRAM} ; \mathrm{HfO}_{2}$; endurance

\section{INTRODUCTION}

Ferroelectricity has received a renewed interest since 2011, with the discovery of a ferroelectric phase in CMOS compatible material $\mathrm{HfO}_{2}$ [1]. Many research studies have been conducted to stabilize the $\mathrm{HfO}_{2}$ orthorhombic oIII-phase with doping, and various dopant incorporation techniques have been proposed [2]. Among them, alternate deposition of $\mathrm{SiO}_{2} / \mathrm{HfO}_{2}$ layers with Atomic Layer Deposition (ALD) is currently the most established doping technique [2]-[4] but is expected to face some limits for ultra-thin layers. Recently, Si doping with Ion Implantation (II) has shown promising ferroelectric performance in a wide range of $\mathrm{Si}$ content [5]. In this paper, a comparison between both doping techniques is proposed for various $\mathrm{Si}$ content and their impact on ferroelectric behavior along field cycling is discussed. Switching kinetics and performance are then investigated down to ultra-short durations for memory applications.

\section{DEVICES}

$100 \times 100 \mu \mathrm{m}^{2} \mathrm{TiN} / \mathrm{HfO}_{2}: \mathrm{Si}(10 \mathrm{~nm}) / \mathrm{TiN}$ capacitances have been fabricated on $300 \mathrm{~mm}$ Si substrates, either by ALD with different $\mathrm{HfO}_{2} / \mathrm{SiO}_{2}$ cycle ratios, or Ion Implantation (II) at $4 \mathrm{keV}$ in ALD-deposited $\mathrm{HfO}_{2}$ layers (Fig.1). For all wafers, anneal at $750^{\circ} \mathrm{C}$ during $30 \mathrm{~s}$ in $\mathrm{N}_{2}$ atmosphere has been performed after TiN top electrode deposition.

While $\% \mathrm{Si}$ in ALD-doped layers is usually calculated as $\mathrm{SiO}_{2} /\left(\mathrm{HfO}_{2}+\mathrm{SiO}_{2}\right)$ cycle ratio, a comparable $\% \mathrm{Si}$ extraction methodology is missing for Si-implanted layers. In order to draw a clear comparison between doping techniques, TOF-SIMS have been performed on 6 dedicated $\mathrm{TiN} / \mathrm{HfO}_{2}: \mathrm{Si}(10 \mathrm{~nm}) / \mathrm{Si}$ fullsheet wafers Fig. 2. Attention has been paid to properly address the different doping profiles obtained in ALD and II cases when extracting the mean $\mathrm{Si}$ concentration, and $\% \mathrm{Si}$ is then calculated as $\% \mathrm{Si}=[\mathrm{Si}]_{\mathrm{avg}} /\left([\mathrm{Si}]_{\mathrm{avg}}+\left[\mathrm{HfO}_{2}\right]_{\text {theoretical }}\right)$. Doping conditions and $\% \mathrm{Si}$ extracted from TOF-SIMS are listed in Table 1.

\section{MATERIAL ANALYSIS}

The ferroelectric properties of Si-doped layers have been extracted by Positive-Up-Negative-Down technique (PUND) along field cycling, as pictured in Fig.3. Fig. 4 (a) shows an example of different PUND I-E characteristics along cycling, with typical ferroelectric peaks and Fig. 4 (b) are the corresponding $\mathrm{P}-\mathrm{E}$ from which the remnant polarization $2 \cdot \mathrm{P}_{\mathrm{R}}=$ $\left|\mathrm{P}_{\mathrm{R}}{ }^{+}\right|+\left|\mathrm{P}_{\mathrm{R}}\right|$ and the mean coercive field $\mathrm{E}_{\mathrm{C}}=\left(\left|\mathrm{E}_{\mathrm{C}}{ }^{+}\right|+\left|\mathrm{E}_{\mathrm{C}}{ }^{-}\right|\right) / 2$ are systematically extracted. Fig.5 summarizes the evolution of ferroelectric parameters along field cycling. All devices are ferroelectric and show an equivalent behavior: remnant polarization increases in all cases (wake-up effect [6]), while a slight decrease of Ec (known as imprint [7]) is observed regardless the doping technique. Both phenomena are usually explained by a redistribution of oxygen vacancies [3], [4], [6] or field-cycling-induced phase transitions [8]. Grazing Incidence X-Ray Diffraction (GIXRD) have been performed and confirm the presence of orthorhombic oIII-phase in both ALD and II case (Fig. 6).

2. $P_{R}$ after $10^{4}$ field cycles, i.e. after the major wake-up of the device, are plotted as a function of \%Si in Fig. 7. Polarization with II is comparable to ALD, thus demonstrating the efficiency of Silicon implant to induce ferroelectricity. II exhibits similar 2. $\mathrm{P}_{\mathrm{R}}$ values for lower $\% \mathrm{Si}$. It is shown that a fine tuning of $\% \mathrm{Si}$ leads to $2 . \mathrm{P}_{\mathrm{R}}$ ranging from 10 up to $18 \mu \mathrm{C} / \mathrm{cm}^{2}$. Fig. 8 represents Cycle-to-BreakDown (CBD) distributions, i.e. the last measured cycle before electrical breakdown. For equivalent polarization values, II devices break slightly before the ALD ones. This is attributed to a higher concentration of process-induced defects with implantation.

\section{SWITCHING KINETICS AND APPLICATION TO MEMORY DEVICES}

The ferroelectric parameters acquired up to now are not representative of realistic memory operations because of the notoptimized field cycling signal used for waking-up ferroelectric layers. In order to address memory performance, electrical switching signals optimization with memory-compatible timings is required. Fig. 9 represents the electrical sequence used to evaluate the switching efficiency of a square pulse with $\mathrm{tr} / \mathrm{tf}=100 \mathrm{~ns}$. Due to the destructive nature of polarization reading operation, a differential approach is proposed, in which the device is set to a known state, followed by the square pulse of interest, aiming to switch device polarization in the opposite state. PUND-like signals are then applied in the same direction 
in order to extract the remaining polarization, i.e. the polarization not switched by the square pulse. Resulting switched polarization percentage have been acquired for various pulse amplitudes and durations, after $10^{4}$ field cycles performed at $4 \mathrm{~V} / 100 \mathrm{kHz}$. Switching efficiency maps of ALD 30:1 and II $3,0 \mathrm{e} 14 \mathrm{at} / \mathrm{cm}^{2}$ are plotted in Fig. 10. It is worth noting that no significant impact of \% $\mathrm{Si}$ is observed within doping technique (not shown here).While showing equivalent static ferroelectric performance (Fig. 5), ALD doping leads to slightly more efficient kinetics at short durations, demonstrating here a $75 \%$ switching capability down to 30ns (i.e. setup limit), compared to 100 ns with II. Both techniques thus appear compatible with memory applications. Moreover, this sequence is suitable for multi-level cell memories as it clearly delimits multiple polarization values depending on the test conditions, i.e. the possibility to achieve more than 2 binary states with a single cell.

From Fig. 10, 5 endurance conditions have been selected and applied to at least 10 capacitances on ALD 30:1 and II 3,0e14 $\mathrm{at} / \mathrm{cm}^{2}$. Contrary to previous field cycling results in which polarization is extracted by $\pm 4 \mathrm{~V}$ PUND technique, an approach equivalent to Fig.9 has been developed in order to extract the realistic switched polarization $\mathrm{P}_{\mathrm{SW}}$ along endurance, i.e. the Memory Window from a memory-application perspective. Fig. 11 shows the impact of pulse parameters on the endurance performance of ALD and II. While both techniques exhibit equivalent behavior, pulse parameters is shown to strongly impact $P_{S w}$, leading to polarization values higher than $2 . P_{R}$ previously extracted along field cycling. In order to explain such difference, specific endurance measurements have been performed with $3 \mathrm{~V} / 1 \mu \mathrm{s}$ square pulses with varying $\mathrm{tr} / \mathrm{tf}$, thus covering both endurance signals $(\mathrm{tr} / \mathrm{tf}=100 \mathrm{~ns})$ and field cycling signals $(\mathrm{tr} / \mathrm{tf}=25 \mu \mathrm{s})$ (Fig. 12). Device wake-up appears to be favored by fast tr/tf, partially explaining the difference between triangular field cycling and square pulse endurance.

Fig.13 summarizes CBD results for the different endurance conditions. As previously noticed, ALD shows better reliability than II, probably due to II-induced defects during device fabrication. In the meanwhile, endurance performance seems to be mainly governed by pulse amplitude, leading to cycling capability as high as $10^{9}$ for both doping techniques when dealing with low-voltage pulses. Considering only $3 \mathrm{~V}$ operations at either $100 \mathrm{~ns}, 1 \mu \mathrm{s}$ or $10 \mu \mathrm{s}$, CBD differences do not scale with pulse durations, demonstrating that $\mathrm{CBD}$ is not Charge-to-Breakdown-related (QBD). Fig.14 shows that CBD correlates with the maximum $\mathrm{P}_{\mathrm{SW}}$ measured during endurance, and a clear trend is established between Memory Window and endurance performance of future memory devices, regardless the doping technique.

Endurance performance of devices with various \%Si have been evaluated with optimized pulse of $3 \mathrm{~V} / 1 \mu \mathrm{s}(\mathrm{tr} / \mathrm{tf}=100 \mathrm{~ns})$. Fig.15 illustrates the evolution of $\mathrm{P}_{\mathrm{Sw}}$ along cycling and brings same qualitative conclusions than field-cycling experiments, i.e. slightly higher polarization performance for ALD and polarization increase with $\% \mathrm{Si}$. CBD achieved with $3 \mathrm{~V} / 1 \mu \mathrm{s}$ square cycling is slightly increased of a factor $\approx 2$ to 3 compared to Fig. 7 with field-cycles at $\pm 4 \mathrm{~V} / 100 \mathrm{kHz}$ (data not shown). On the basis of these tests, $\mathrm{P}_{\mathrm{SW}}{ }^{\max }$ as a function of CBD is plotted in Fig. 16. It is shown that, besides electrical signal engineering, a fine tuning of $\% \mathrm{Si}$ allows to improve the CBD / Memory
Window compromise, best results being obtained for 24:1 ALD doping technique.

\section{CONCLUSION}

For the first time, a thorough comparison between ALD and II doping techniques in ferroelectric Si-doped $\mathrm{HfO}_{2}$ have been established. II has shown to exhibit equivalent ferroelectric behavior compared to ALD (wake-up and imprint), equivalent polarization performance at lower $\% \mathrm{Si}$, and slightly lower cycling capability, attributed to process-induced defects. Further optimization of the II doping technique is thus as promising as the common ALD doping technique for memory application.

Thanks to an electrical test method, switching kinetics have been evaluated for various process conditions, showing a 30ns switching capability for ALD and 100ns for II. The ferroelectric behavior is tunable through the pulse's amplitude, width and tr/tf. Subsequent endurance results have shown that proper signal engineering allows to finely tune the CBD / Memory Window compromise demonstrating an endurance capability as high as $10^{9}$ for both doping techniques.

\section{ACKNOWLEDGMENT}

This work have been partially supported by the European WakeMeUp project.

\section{REFERENCES}

[1] T. S. Böscke, J. Müller, D. Bräuhaus, U. Schröder, and U. Böttger, 'Ferroelectricity in hafnium oxide thin films', Appl. Phys. Lett., vol. 99, no. 10, p. 102903, Sep. 2011.

[2] M. H. Park et al., 'Ferroelectricity and Antiferroelectricity of Doped Thin $\mathrm{HfO}_{2}$-Based Films', Adv. Mater., vol. 27, no. 11, pp. 1811-1831, Mar. 2015.

[3] C. Richter et al., 'Si Doped Hafnium Oxide-A "Fragile" Ferroelectric System', Adv. Electron. Mater., vol. 3, no. 10, p. 1700131, Oct. 2017.

[4] M. H. Park, Y. H. Lee, T. Mikolajick, U. Schroeder, and C. S. Hwang, 'Review and perspective on ferroelectric $\mathrm{HfO} 2$-based thin films for memory applications', MRS Commun., vol. 8, no. 03, pp. 795-808, Sep. 2018.

[5] T. Francois et al., 'Evaluation of Ferroelectricity in Si-implanted $\mathrm{HfO}_{2}$ along Cycling', SSDM 2018 proceedings, 2018.

[6] M. Pešić et al., 'Physical Mechanisms behind the Field-Cycling Behavior of $\mathrm{HfO}_{2}$-Based Ferroelectric Capacitors', Adv. Funct. Mater., vol. 26, no. 25, pp. 4601-4612, Jul. 2016.

[7] F. P. G. Fengler, M. Hoffmann, S. Slesazeck, T. Mikolajick, and U. Schroeder, 'On the relationship between field cycling and imprint in ferroelectric $\mathrm{Hf}_{0.5} \mathrm{Zr}_{0.5} \mathrm{O}_{2}$ ', J. Appl. Phys., vol. 123, no. 20, p. 204101, May 2018.

[8] P. D. Lomenzo et al., 'TaN interface properties and electric field cycling effects on ferroelectric Si-doped $\mathrm{HfO}_{2}$ thin films', J. Appl. Phys., vol. 117, no. 13, p. 134105, Apr. 2015. 


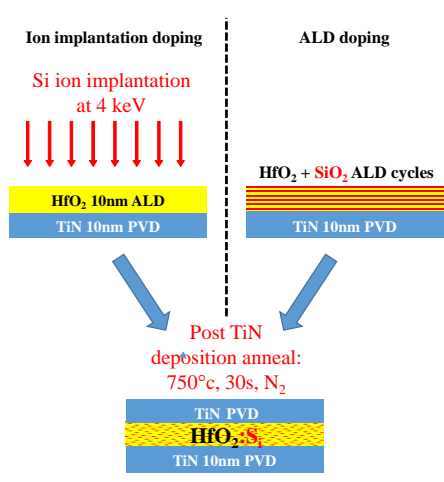

Fig. 1: Schematic representation of both doping techniques to achieve $100 \times 100 \mu \mathrm{m}^{2}$ capacitances on $300 \mathrm{~mm}$ wafers. All the other steps of the process flow are strictly the same.

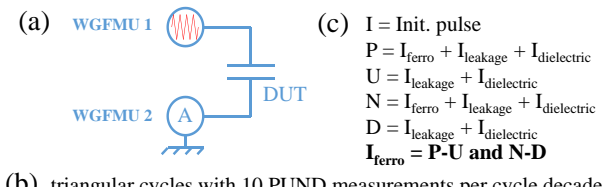

(b) triangular cycles with 10 PUND measurements per cycle decade

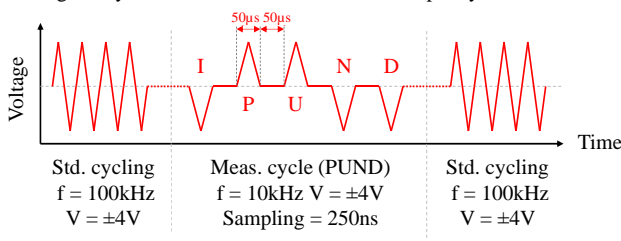

Fig. 3: (a) Schematic representation of measurement setup, based on 2 Keysight B1530 Waveform Generator/Fast Measurement Units (WGFMU) (b) Applied test sequence: triangular waking-up cycles $( \pm 4 \mathrm{~V} / 100 \mathrm{kHz})$ with PUND $( \pm 4 \mathrm{~V} / 10 \mathrm{kHz})$ ferroelectric measurements performed 10 times per cycle decade. (c) PUND measurement description for correcting leakage and dielectric displacement current contributions.

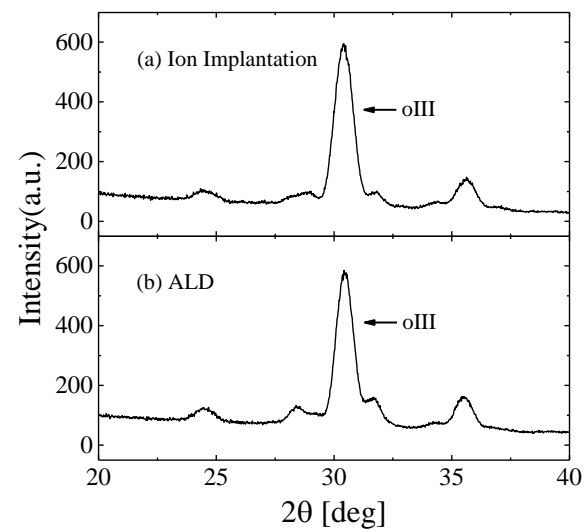

Fig. 6: GIXRD measurement of (a) Ion Implantation $3,4 \mathrm{e} 14 \mathrm{at} / \mathrm{cm}^{2}$ and (b) ALD 30:1. The ferroelectric orthorhombic oIII-phase peaks confirm the electrical measurement for both doping techniques.

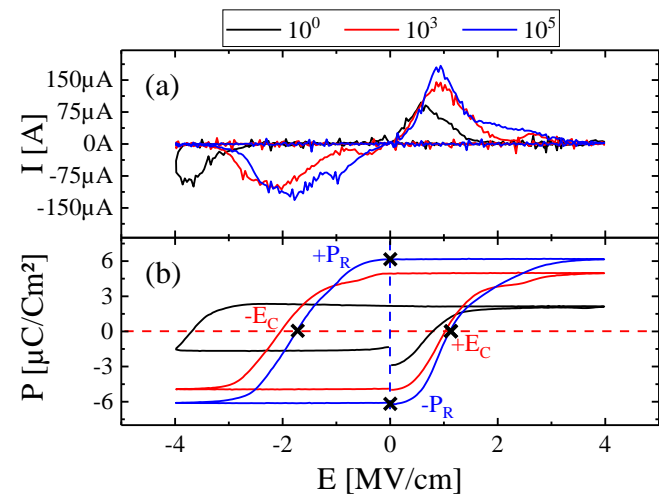

Fig. 4: For the ALD case 30:1 2,38\% Si, (a) I-E at (black) $10^{0}$, (red) $10^{3}$ and (blue) $10^{5}$ cycles obtained by PUND technique where typical ferroelectric peaks are visible and (b) corresponding P-E with the ferroelectric metrics, i.e. $\mathrm{P}_{\mathrm{R}}{ }^{+-}$and $\mathrm{E}_{\mathrm{C}}{ }^{+/-}$, highlighted by the marks.

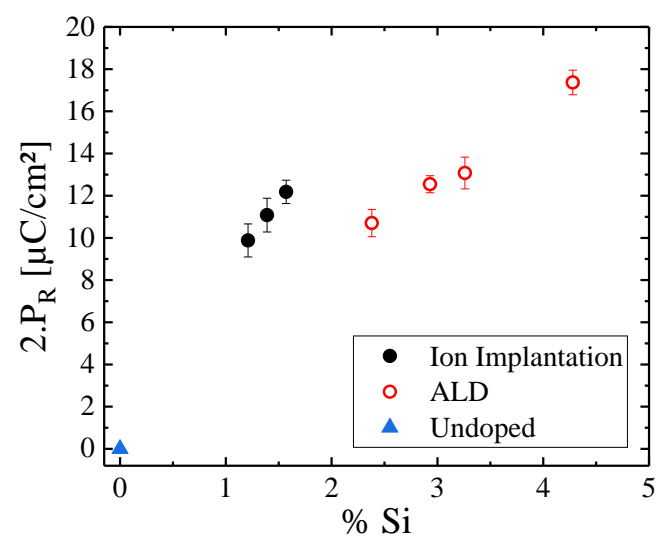

Fig. 7: $2 . P_{R}$ after $10^{4}$ wake-up cycles at $4 \mathrm{~V} / 100 \mathrm{kHz}$ measured by a PUND signal at $4 \mathrm{~V} / 10 \mathrm{kHz}$ as a function of $\%$ Si for both technologies.

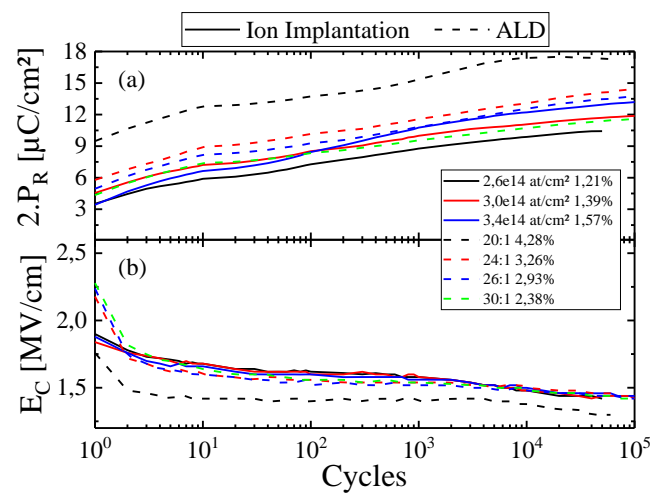

Fig. 5: (a) median 2.P $P_{R}$ and (b) median $E_{C}$ along triangular wake-up cycling $4 \mathrm{~V} / 100 \mathrm{kHz}$ for all doping conditions extracted 10 times by decade by a PUND read signal at $4 \mathrm{~V} / 10 \mathrm{kHz}$. Straight lines correspond to II while dashed lines are for ALD.

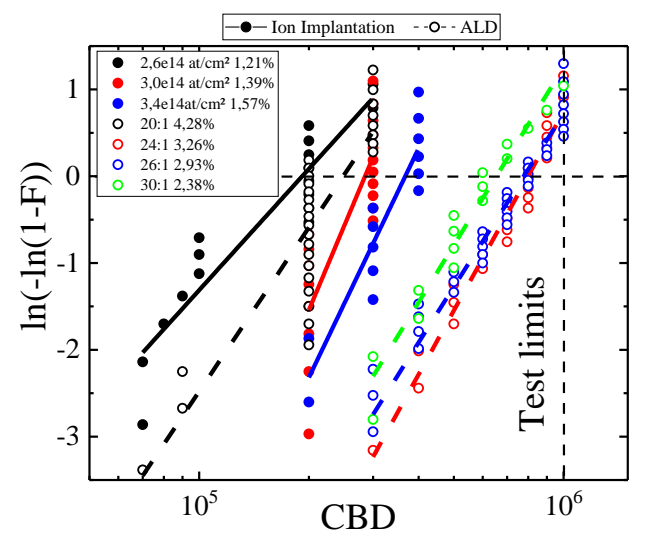

Fig. 8: CBD distribution of all doping conditions under wake-up triangular signal $4 \mathrm{~V} / 100 \mathrm{kHz}$ up to $10^{6}$ cycles. 


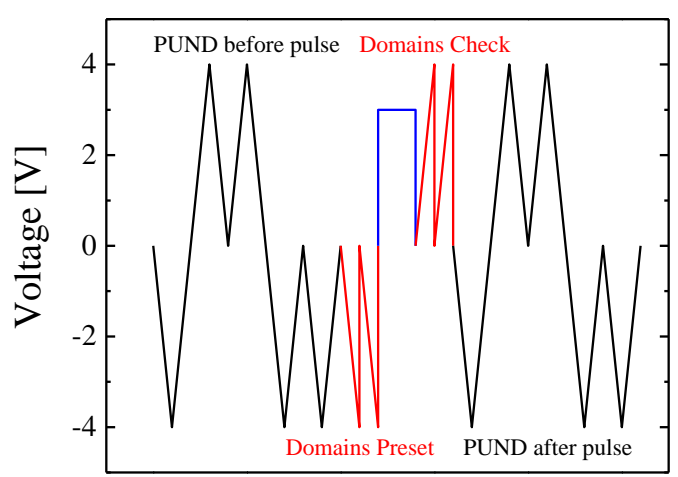

Time

Fig. 9: Sequence used, after $10^{4}$ wake-up signal $4 \mathrm{~V} / 100 \mathrm{kHz}$, to evaluate the switching efficiency of square pulses. The PUND $4 \mathrm{~V} / 10 \mathrm{kHz}$ are used as polarization references. The domains preset signal ensure the initialization of the opposite state, while the domains check signal measure the polarization which has not been switched by the pulse. Both checks are double ramp to perform a PUND-like extraction, cf. Fig. 3 (c).

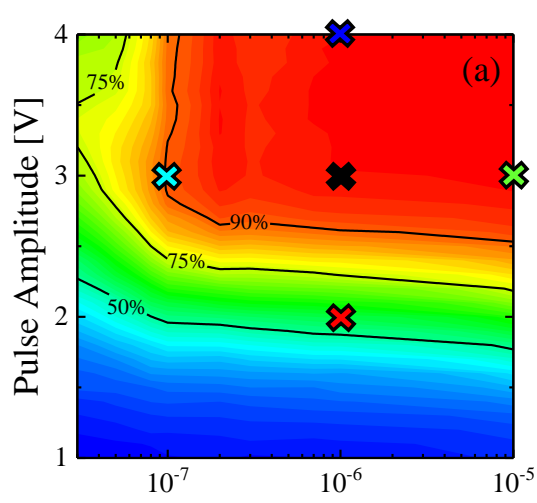

Pulse Width [s]

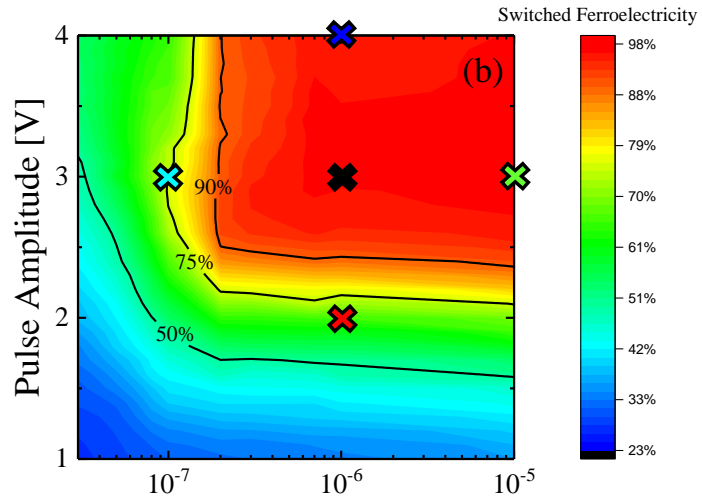

Pulse Width [s]

Fig. 10: Switching efficiency maps of (a) ALD 30:1 2,38\% and (b) II 3,0e14 at/ $/ \mathrm{cm}^{2} 1,39 \%$ with tr/tf $=100 \mathrm{~ns}$. These devices have the same behavior along wake-up cycling according to Fig.5 (a). The marks indicate the chosen parameters for the following study.

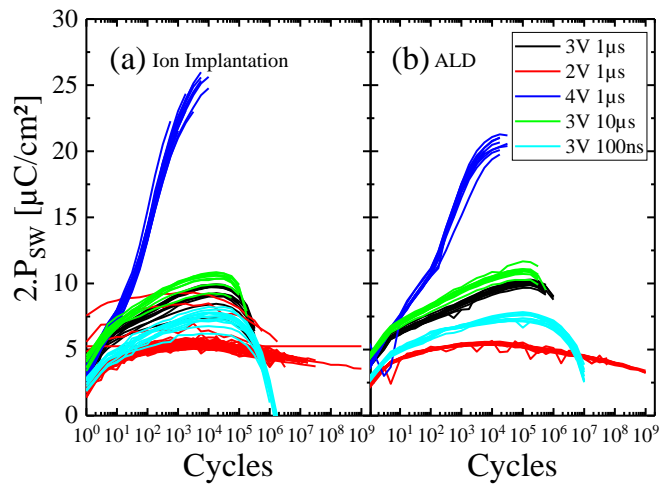

Fig.11: 2.Psw along cycling of (a) II 3,0e14 at $/ \mathrm{cm}^{2} 1,39 \%$ and (b) ALD 30:1 2,38\% with 5 different pulse conditions and $\mathrm{tr} / \mathrm{tf}=100 \mathrm{~ns}$.

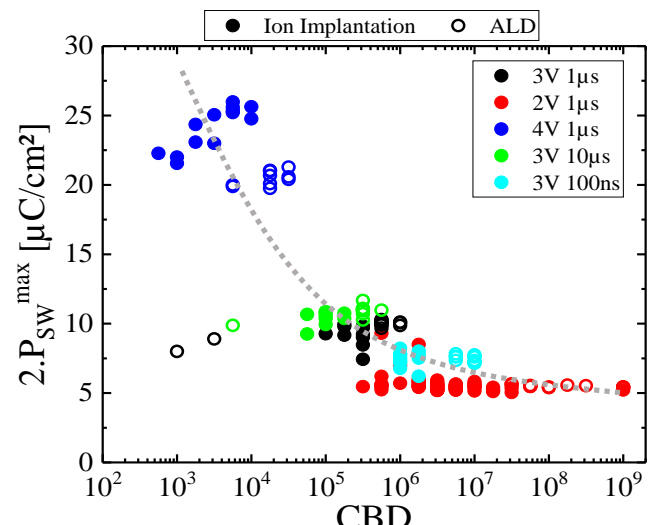

Fig. 14: 2.P $\mathrm{P}_{\mathrm{Sw}}{ }^{\max }$ achieved during square cycling with various conditions and $\mathrm{tr} / \mathrm{tf}_{1}=100 \mathrm{~ns}$ as a function of the corresponding die's CBD. Tested devices are ALD 30:1 $2,38 \%$ and II $3,0 \mathrm{e} 14$ at $/ \mathrm{cm}^{2} 1,39 \%$. The trend is highlighted by the dashed line.

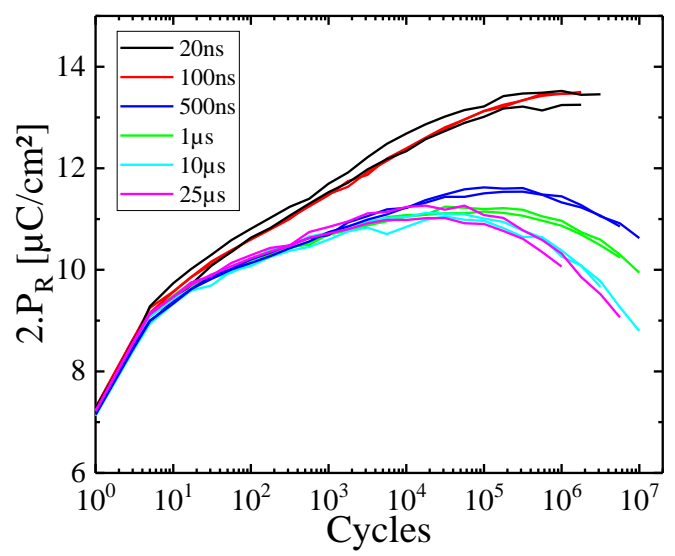

Fig. 12: Impact of the tr/tf along cycling for $24: 13,26 \%$ case. The pulse parameters are $3 \mathrm{~V} / 1 \mu \mathrm{s}$ for every measure.

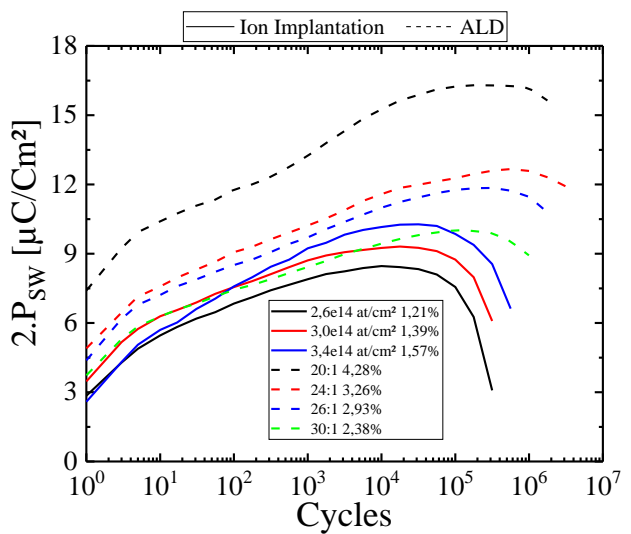

Fig. 15: Median 2.P Sw $_{\text {along optimized square cycling }}$ $3 \mathrm{~V} / 1 \mu \mathrm{s}$ and $\mathrm{tr} / \mathrm{tf}=100 \mathrm{~ns}$ for all doping conditions extracted 5 times by decade.

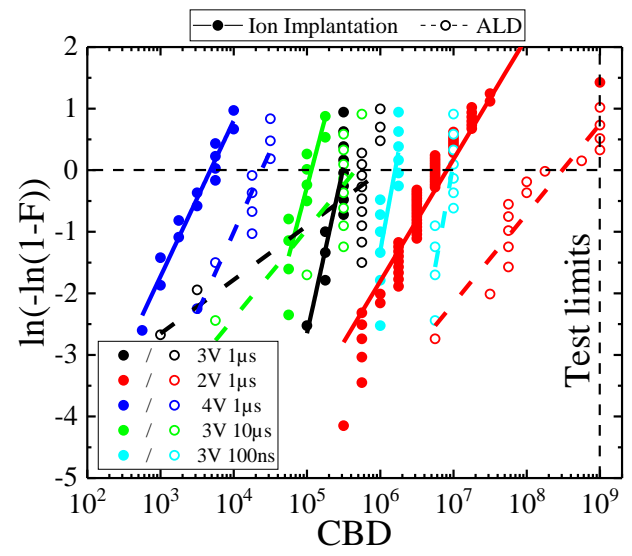

Fig. 13: CBD distribution of ALD 30:1 2,38\% and II $3,0 \mathrm{e} 14 \mathrm{at} / \mathrm{cm}^{2} 1,39 \%$ with 5 different pulse conditions and $\mathrm{tr} / \mathrm{tf}=100 \mathrm{~ns}$.

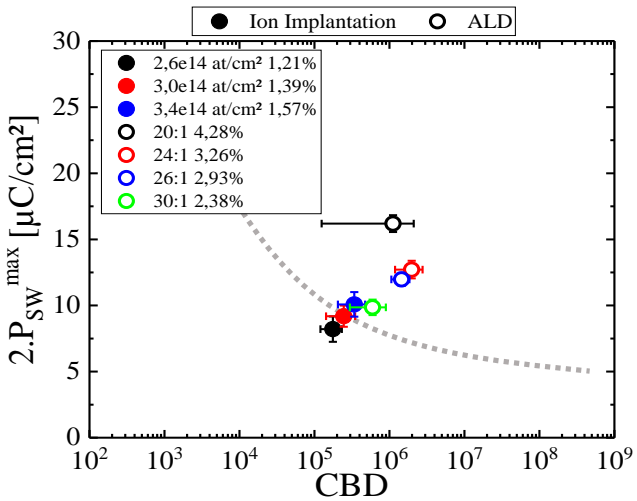

Fig. 16: $2 . \mathrm{P}_{\mathrm{SW}}{ }^{\max }$ achieved during optimized square cycling, $3 \mathrm{~V} / 1 \mu \mathrm{s}$ and $\mathrm{tr} / \mathrm{tf}=100 \mathrm{~ns}$, as a function of the corresponding $\mathrm{CBD}$, for all doping conditions. The dashed line corresponds to the previous trend of Fig. 14. 